\title{
Efficiency at maximum power and efficiency fluctuations in a linear Brownian heat engine model
}

\author{
Jong-Min Park, ${ }^{1}$ Hyun-Myung Chun, ${ }^{1}$ and Jae Dong Noh ${ }^{1,2}$ \\ ${ }^{1}$ Department of Physics, University of Seoul, Seoul 02504, Korea \\ ${ }^{2}$ School of Physics, Korea Institute for Advanced Study, Seoul 02455, Korea
}

(Dated: March 25, 2016)

\begin{abstract}
We investigate stochastic thermodynamics of a two-particles Langevin system. Each particle is in contact with a heat bath at different temperatures $T_{1}$ and $T_{2}\left(<T_{1}\right)$, respectively. Particles are trapped by a harmonic potential and driven by a linear external force. The system can act as an autonomous heat engine performing work against the external driving force. Linearity of the system enables us to examine thermodynamic properties of the engine analytically. We find that the efficiency of the engine at maximum power $\eta_{M P}$ is given by $\eta_{M P}=1-\sqrt{T_{2} / T_{1}}$. This universal form has been known as a characteristic of endoreversible heat engines. Our result extends the universal behavior of $\eta_{M P}$ to non-endoreversible engines. We also obtain the large deviation function of the probability distribution for the stochastic efficiency in the overdamped limit. The large deviation function takes the minimum value at mean efficiency $\eta=\bar{\eta}$ and increases monotonically until it reaches plateaus when $\eta \leq \eta_{L}$ and $\eta \geq \eta_{R}$ with model dependent parameters $\eta_{R, L}$. It has been known for heat engines with a finite number of microscopic configurations with time-symmetric protocol that the probability of achieving the Carnot efficiency is minimum. Our result reveals that the least likeliness of the Carnot efficiency is not the generic property of heat engines.
\end{abstract}

PACS numbers: 05.40.-a, 05.40.Jc, 05.70.Ln

\section{INTRODUCTION}

Heat engines are devices to generate mechanical work by exploiting heat flows between hot and cold heat baths at temperatures $T_{1}$ and $T_{2}\left(<T_{1}\right)$. Since the advance of stochastic thermodynamics, Brownian heat engines consisting of microscopic small components have been attracting a lot of theoretical and experimental interests. Those engines are working in nonequilibrium conditions and subject to large thermal fluctuations. Much efforts have been devoted to understanding common properties that are shared by a variety of different engine models.

The efficiency $\eta$, defined as the ratio of the work to the absorbed heat from a hot heat bath, is one of the most important characteristics of a heat engine. According to the thermodynamic laws, the efficiency is limited from above by the Carnot efficiency $\eta_{C} \equiv 1-T_{2} / T_{1}$. The Carnot efficiency is achieved only when an engine operates infinitely slow and reversibly. Hence, an engine operating at the Carnot efficiency is of no practical importance because its power, work per unit time, is zero.

Instead of optimizing the efficiency, researchers are interested in the efficiency of an engine when it is optimized to yield the maximum power, which is called the efficiency at maximum power (EMP) $\eta_{M P}$. The EMP is shown to be universal for endoreversible engines that operate reversibly except when they exchange heats with external heat baths 1]. The EMP of the endoreversible engines is given by $\eta_{M P}=\eta_{C A} \equiv 1-\sqrt{T_{2} / T_{1}}$. This efficiency $\eta_{C A}$ is called the Curzon-Ahlborn efficiency since it was rediscovered by Curzon and Ahlborn [1] while it was first known long before [2, 3].

Most of realistic engines are not endoreversible [4, 5]. Nevertheless, the EMP of many engines is close to $\eta_{C A}$ when $T_{1}$ and $T_{2}$ are close to each other so that $\eta_{C} \ll 1$. In this limit, the Curzon-Ahlborn efficiency is expanded as $\eta_{C A}=1-\sqrt{1-\eta_{C}}=\frac{1}{2} \eta_{C}+\frac{1}{8} \eta_{C}^{2}+O\left(\eta_{C}^{3}\right)$. Some engines, which are not endoreversible, share the same expansion up to first or second order in $\eta_{C}$ [6-28]. It was found that the first order term reflects the strong coupling between thermodynamic fluxes [29] and that the second order term the left-right symmetry [30]. The universality of the expansion has been investigated in the context of irreversible thermodynamics 31].

When one measures the efficiency of an engine for a time interval $t$, it varies from one measurement to another due to thermal fluctuations. Thus, the efficiency is a fluctuating random variable characterized by the probability distribution function $P_{t}(\eta)$ and the large deviation function $L(\eta) \equiv-\lim _{t \rightarrow \infty} \frac{1}{t} \ln P_{t}(\eta)$ in the long time limit. Recently, it was found that the large deviation function $L(\eta)$ is maximum at $\eta=\eta_{C}$. This means that the Carnot efficiency is least likely in the $t \rightarrow \infty$ limit. To be precise, such a property was proved for a heat engine which has only a finite number of microscopic configurations and is driven by a time-symmetric protocol [32, 33]. The least likeliness of the Carnot efficiency was demonstrated in two-level systems analytically and numerically. However, it remains as an open question whether it is valid for systems with continuous variables.

In this paper, we introduce an exactly solvable model for a Brownian heat engine. The model consists of two Brownian particles in one dimension which are trapped by a harmonic potential and driven by a linear external force. Each particle is in contact with a heat bath at different temperatures. The temperature difference induces a heat flow, which enables the system to work against the external force. Owing to solvability, the lin- 
ear systems have been adopted for detailed study of various subjects in stochastic thermodynamics such as the entropy production, the fluctuation theorems, information engines, and so on [34 37]. We will investigate thoroughly the linear model in the perspective of the heat engine with the focus on the efficiency of the heat engine. Our results can be summarized as follows: (i) The exact expressions for the average efficiency and power are derived. We find that the EMP is equal to $\eta_{C A}$. Our engine model operates in a nonequilibrium condition, hence is not an endoreversible engine. This result indicates that the endoreversibility is not a necessary condition for $\eta_{M P}=\eta_{C A}$. (ii) The large deviation function $L(\eta)$ for the efficiency is obtained analytically. The function is minimum at the average efficiency, increases monotonically as $\eta$ departs from the average efficiency, and reaches constant plateaus in the regions with $\eta \geq \eta_{R}$ and $\eta \leq \eta_{L}$. The large deviation function does not have a peak at the Carnot efficiency, which is in sharp contrast to the property of finite-configurations heat engines.

This paper is organized as follows. We introduce the model system and calculate the steady state average of the heat and work in Sec. III. We elaborate on the EMP and compare it with $\eta_{C A}$ in Sec. III In Sec. IV we derive the exact expression for the large deviation function for the efficiency. We summarize our results in Sec. $\mathbf{D}$

\section{LINEAR ENGINE MODEL}

We consider a system consisting of two Brownian particles of mass $m$ in one dimension. Two particles are in contact with two different heat baths at temperatures $T_{1}$ and $T_{2}\left(<T_{1}\right)$, respectively, and linear forces are applied. Their motions are governed by the underdamped Langevin equations

$$
\begin{aligned}
\dot{x}_{1} & =v_{1}, \\
\dot{x}_{2} & =v_{2}, \\
m \dot{v}_{1} & =-\gamma v_{1}-K x_{1}+\epsilon x_{2}+\xi_{1}(t), \\
m \dot{v}_{2} & =-\gamma v_{2}-K x_{2}+\delta x_{1}+\xi_{2}(t),
\end{aligned}
$$

where $x_{i}$ and $v_{i}$ are the position and the velocity of $i(=1,2)$ th particle, $\gamma$ is a damping coefficient, $K$ is a stiffness constant of a harmonic potential trapping the particles at the origin, $(\epsilon, \delta)$ are the coupling constants, and $\xi_{i}(t)$ is the Gaussian-distributed random force satisfying $\left\langle\xi_{i}(t)\right\rangle=0$ and $\left\langle\xi_{i}(t) \xi_{j}\left(t^{\prime}\right)\right\rangle=2 \gamma k_{B} T_{i} \delta_{i j} \delta\left(t-t^{\prime}\right)$. We use a shorthand notation ' for a time derivative and set the Boltzmann constant $k_{B}$ to be unity hereafter.

The two-particle system may be interpreted as a single Brownian particle system in two dimensions with position column vector $\boldsymbol{x}=\left(x_{1}, x_{2}\right)^{T}$ and velocity column vector $\boldsymbol{v}=\left(v_{1}, v_{2}\right)^{T}=\dot{\boldsymbol{x}}$. The superscript ${ }^{T}$ stands for the transpose. In this interpretation, the total applied force $\boldsymbol{f}$ is decomposed into the sum of two parts: $\boldsymbol{f}=\boldsymbol{f}_{c}+\boldsymbol{f}_{n c}$ with the conservative force

$$
\boldsymbol{f}_{c}=-K \boldsymbol{x}=-\nabla V(\boldsymbol{x})
$$

with a harmonic potential $V(\boldsymbol{x})=\frac{1}{2} K \boldsymbol{x}^{2}$ and the nonconservative driving force

$$
\boldsymbol{f}_{n c}=\left(\epsilon x_{2}, \delta x_{1}\right)^{T}
$$

which does not have a corresponding potential function unless $\epsilon=\delta$. The motions along the $x_{1}$-axis and the $x_{2}$-axis are affected independently by the heat baths of temperatures $T_{1}$ and $T_{2}$, respectively.

For appropriate choices of $\epsilon$ and $\delta$, the system can work against the nonconservative force by exploiting the heat flow between the heat baths. Thus it can act as a heat engine as well as a heat pump or a refrigerator. According to stochastic energetics [38], the heats absorbed from the heat baths into the system and the work done by the system against the driving force during an infinitesimal time interval $[t, t+d t]$ are given by

$$
\begin{aligned}
& d Q_{1}(t)=v_{1}(t) \circ\left[-\gamma v_{1}(t) d t+d \Xi_{1}(t)\right] \\
& d Q_{2}(t)=v_{2}(t) \circ\left[-\gamma v_{2}(t) d t+d \Xi_{2}(t)\right] \\
& d W(t)=-\boldsymbol{f}_{n c} \circ d \boldsymbol{x}=-\left[\epsilon v_{1}(t) x_{2}(t)+\delta x_{1}(t) v_{2}(t)\right] d t
\end{aligned}
$$

where $d \Xi_{i}(t) \equiv \int_{t}^{t+d t} d t^{\prime} \xi_{i}\left(t^{\prime}\right)$ are Gaussian random variables satisfying $\left\langle d \Xi_{i}(t)\right\rangle=0$ and $\left\langle d \Xi_{i}(t) d \Xi_{j}(t)\right\rangle=$ $2 \gamma T_{i} \delta_{i j} d t$. The notation o represents the Stratonovich product [39, 40]. Those quantities satisfy the energy conservation $d E(t)=d Q_{1}(t)+d Q_{2}(t)-d W(t)$ with the internal energy $E=\frac{1}{2} m \boldsymbol{v}^{2}+V(\boldsymbol{x})$.

We focus on the average quantities in the steady state, denoted by $\langle\cdot\rangle_{s}$. Fluctuations are considered later. The steady-state average of the internal energy change, $\langle d E\rangle_{s}$, vanishes. Hence, there exist only two relevant quantities describing the energy flow. We choose the heat flow rate from the hot reservoir $q_{1} \equiv\left\langle d Q_{1} / d t\right\rangle$ and the work production rate $w \equiv\langle d W / d t\rangle_{s}$. The Stratonovich algebra yields that $\left\langle v_{1}(t) \circ d \Xi_{1}(t)\right\rangle_{s}=\left\langle\frac{1}{2}\left(v_{1}(t)+v_{1}(t+\right.\right.$ $\left.d t)) d \Xi_{1}(t)\right\rangle_{s}=\frac{\gamma T_{1}}{m} d t+o(d t)$. Thus, we obtain the expressions [37, 41 43 .

$$
\begin{aligned}
q_{1} & =\frac{2 \gamma}{m}\left(\frac{T_{1}}{2}-\frac{1}{2} m\left\langle v_{1}^{2}\right\rangle_{s}\right), \\
w & =-\epsilon\left\langle v_{1} x_{2}\right\rangle_{s}-\delta\left\langle x_{1} v_{2}\right\rangle_{s} .
\end{aligned}
$$

The average heat flux from the cold reservoir is given by $q_{2} \equiv\left\langle d Q_{2} / d t\right\rangle_{s}=w-q_{1}$.

The Langevin equations in (11) are linear in $\boldsymbol{z}=$ $\left(x_{1}, x_{2}, v_{1}, v_{2}\right)^{T}$ and belong to the class of the multivariate Ornstein-Uhlenbeck process [39, 40]. In such a case, the steady state is Gaussian-distributed with the covariance matrix $\Sigma=\left\langle\boldsymbol{z} \boldsymbol{z}^{T}\right\rangle_{s}$ being determined as a solution of a set of linear equations. Following the standard procedure (see Sec.4.5.6 of Ref. [40]), we obtain that

$$
\Sigma=\left(\begin{array}{cccc}
\frac{\left(K \psi+\gamma^{2} \phi\right)}{\delta} & \psi & 0 & \gamma \phi \\
\psi & \frac{\left(K \psi-\gamma^{2} \phi\right)}{\epsilon} & -\gamma \phi & 0 \\
0 & -\gamma \phi & \frac{T_{1}}{m}-\epsilon \phi & 0 \\
\gamma \phi & 0 & 0 & \frac{T_{2}}{m}+\delta \phi
\end{array}\right)
$$




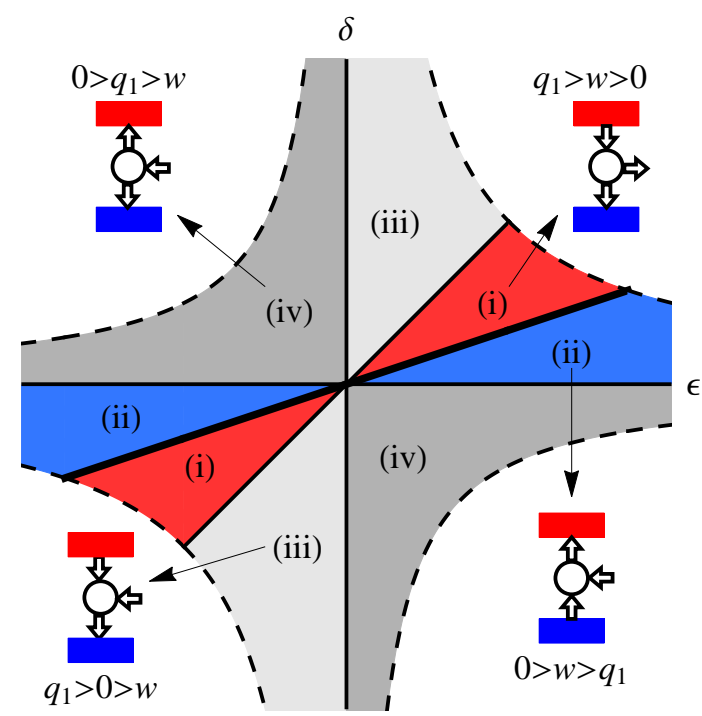

FIG. 1. Function diagram of the linear engine model. The dashed lines are the boundary of the stable region.

with $\psi=\frac{\delta T_{1}+\epsilon T_{2}}{2\left(K^{2}-\epsilon \delta\right)}$ and $\phi=\frac{\delta T_{1}-\epsilon T_{2}}{2\left(\gamma^{2} K+m \epsilon \delta\right)}$. Using the covariance matrix, we find that

$$
\begin{aligned}
& q_{1}=\gamma \epsilon \phi=\frac{\gamma \epsilon\left(\delta T_{1}-\epsilon T_{2}\right)}{2\left(\gamma^{2} K+m \epsilon \delta\right)} \\
& w=\gamma(\epsilon-\delta) \phi=\frac{\gamma(\epsilon-\delta)\left(\delta T_{1}-\epsilon T_{2}\right)}{2\left(\gamma^{2} K+m \epsilon \delta\right)} .
\end{aligned}
$$

The covariance matrix is positive-definite in the region

$$
-\frac{\gamma^{2} K}{m}<\epsilon \delta<K^{2}
$$

Outside the region, the nonconservative force is so strong that the particle escapes from the harmonic potential. We will restrict ourselves to the stable region for further analysis.

\section{EFFICIENCY AT MAXIMUM POWER}

As one varies $\epsilon$ and $\delta$ within the stable region, $q_{1}$ and $w$ flip their signs. There are four different regions: (i) When $q_{1}>0$ and $w>0$, the system operates as a heat engine which absorbs a heat from the hot bath, dissipates a heat to a cold bath, and works against the driving force. The average engine efficiency is given by

$$
\bar{\eta}=\frac{w}{q_{1}}=1-\frac{\delta}{\epsilon} .
$$

We use the notation $\bar{\eta}$ for the average efficiency in order to distinguish it from the stochastic efficiency $\eta$ investigated later. (ii) When $q_{1}<0, w<0$ and $q_{2}=w-q_{1}>0$, the system operates as a heat pump or a refrigerator which transfers a heat from the cold bath $\left(q_{2}\right)$ to the hot bath $\left(\left|q_{1}\right|\right)$ with the help of an external work $(|w|)$.

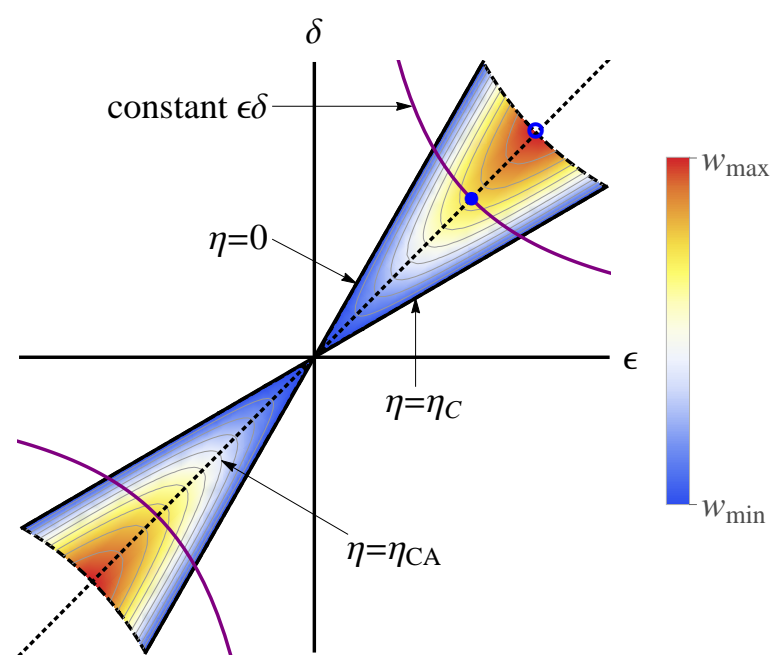

FIG. 2. Density and contour plots for the engine power $w$.

(iii) When $q_{1}>0, w<0$, and $q_{2}<0$, a heat flows from the hot bath to the cold bath at the expense of an external work. (iv) When $q_{1}<0, w<0$ and $q_{2}<0$, an external work is dissipated into the two baths. The border lines of these four regions and the stable region are drawn in Fig. 1. The two regions (iii) and (iv) are of no practical importance. We focus on the heat engine region (i).

The regions (i) and (iii) are separated by the line $\delta=\epsilon$, where the force $\boldsymbol{f}_{n c}$ becomes a conservative one. Hence the power $w$ vanishes and the system plays a role of a heat conductor.

The heat engine regime (i) is separated from the heat pump regime (ii) by the line $\delta=\left(T_{2} / T_{1}\right) \epsilon=\left(1-\eta_{C}\right) \epsilon$, drawn with the thick line in Fig. 1 Along this line, the efficiency in (9) is given by the Carnot efficiency $\eta_{C}=1-T_{2} / T_{1}$ with vanishing power (see (7)). In macroscopic thermodynamics, the Carnot efficiency is achieved only when an engine operates quasi-statically and reversibly. The vanishing power and the Carnot efficiency along the line are thus consistent with each other [6, 8, 12]. In fact, our model can be shown to be in thermal equilibrium along the line $\delta=\left(1-\eta_{C}\right) \epsilon$. In terms of dimensionless parameters $\tilde{x}_{1}=\frac{x_{1}}{\left(\sqrt{m T_{1}} / \gamma\right)}$, $\tilde{x}_{2}=\frac{x_{2}}{\left(\sqrt{m T_{2}} / \gamma\right)}$, and $\tilde{t}=\frac{t}{(m / \gamma)}$, the Langevin equation (1) becomes equivalent to that for a two-dimensional Brownian particle in thermal contact with a single heat bath at unit temperature. The particle is driven by the effective nonconservative force $\tilde{\boldsymbol{f}}_{n c}=\left(\frac{m}{\gamma^{2}} \sqrt{\frac{T_{2}}{T_{1}}} \epsilon \tilde{x}_{2}, \frac{m}{\gamma^{2}} \sqrt{\frac{T_{1}}{T_{2}}} \delta \tilde{x}_{1}\right)^{T}$, which turns into the conservative force along the line $\delta=\left(T_{2} / T_{1}\right) \epsilon=\left(1-\eta_{C}\right) \epsilon$. The temperature difference $\left(T_{1} \neq T_{2}\right)$ and the nonconservative force $f_{\mathrm{nc}}$ are the ingredients that drive the system out of equilibrium. When $\delta T_{1}=\epsilon T_{2}$, their effects cancel each other and the system is in thermal equilibrium.

The power $w$ of the engine varies in the $(\epsilon, \delta)$ plane as shown in Fig. 2] We will find the maximum power point 
and investigate how the EMP depends on the temperatures. The power $w$ is given by a function of $\epsilon$ and $\delta$ in (77). Recalling that the average efficiency $\bar{\eta}$ in (9) is a function of $\delta / \epsilon$, we found it convenient to write $w$ as a function of $\epsilon \delta$ and $\bar{\eta}$ instead of a function of $\epsilon$ and $\delta$ :

$$
w(\epsilon \delta, \bar{\eta})=\frac{\gamma \epsilon \delta T_{1}}{2\left(\gamma^{2} K+m \epsilon \delta\right)} \frac{\bar{\eta}\left(\eta_{C}-\bar{\eta}\right)}{1-\bar{\eta}}
$$

with $\eta_{C}=1-T_{2} / T_{1}$. Then, for a given $\epsilon \delta$, the power is maximum when $\frac{\partial w}{\partial \bar{\eta}}=0$, which yields that

$$
\eta_{M P}=1-\sqrt{1-\eta_{C}} .
$$

This is the EMP along the constant- $\epsilon \delta$ curves (see Fig. (2). The global maximum of the power is achieved in the limiting case where $\epsilon \delta$ approaches $K^{2}$, the border of the stable region (see (8)), the efficiency at which is also given by (11).

To our surprise, the result for the efficiency at maximum power is the same as the Curzon-Ahlborn efficiency $\eta_{C A}$ obtained for the endoreversible engine [1]. It reveals that the endoreversibility is not a necessary condition for $\eta_{M P}=\eta_{C A}$. In order to understand the similarity between our model and the endoreversible engines, we rederive the Curzon-Ahlborn result [1, 44, 45]. An endoreversible engine operates under the assumption that it maintains internal temperatures $T_{1 i}$ and $T_{2 i}$ when it exchanges heats with the heat baths at temperatures at $T_{1}$ and $T_{2}$, respectively. The endoreversibility means that the engine operates as the Carnot engine between two temperatures $T_{1 i}$ and $T_{2 i}$. Assuming the Fourier law, the incoming $\left(q_{1}\right)$ and outgoing $\left(-q_{2}\right)$ heat fluxes are given by $q_{1}=\alpha_{1}\left(T_{1}-T_{1 i}\right)$ and $-q_{2}=\alpha_{2}\left(T_{2 i}-T_{2}\right)$, respectively, with the heat conductivities $\alpha_{i}$. Then, the endoreversible condition amounts to $q_{1} / T_{1 i}=-q_{2} / T_{2 i}$. The power is given by $w=q_{1}+q_{2}=\alpha_{1}\left(T_{1}-T_{1 i}\right)-\alpha_{2}\left(T_{2 i}-T_{2}\right)$. It is a function of the internal temperatures $T_{1 i}$ and $T_{2 i}$ which are determined by an operating condition. Using the endoreversible condition and the expression for the efficiency $\bar{\eta}=1-\frac{\alpha_{2}\left(T_{2 i}-T_{2}\right)}{\alpha_{1}\left(T_{1}-T_{1 i}\right)}$, one can eliminate $T_{1 i}$ and $T_{2 i}$ in $w$ to obtain that

$$
w=\frac{\alpha_{1} \alpha_{2} T_{1}}{\alpha_{1}+\alpha_{2}} \frac{\bar{\eta}\left(\eta_{C}-\bar{\eta}\right)}{1-\bar{\eta}}
$$

Apart from the overall factor, it has the same $\bar{\eta}$ dependence as in (10), hence the same efficiency at maximum power.

Comparing (10) and (12), one finds that the CurzonAhlborn efficiency $\eta_{C A}=1-\sqrt{T_{2} / T_{1}}$ is the consequence of the specific relation between the thermodynamic quantities irrespective of microscopic details of engines. It is convenient to use the parameters $s_{1}=q_{1} / T_{1}$ (entropy loss of the hot bath) and $s_{2}=-q_{2} / T_{2}$ (entropy gain of the cold bath). Using $w=s_{1} T_{1}-s_{2} T_{2}$ and $\bar{\eta}=1-\left(s_{2} T_{2}\right) /\left(s_{1} T_{1}\right)$, we can rewrite (10) and (12) in the form $s_{1}=\mathcal{F}\left(s_{2}\right)$ where the function $\mathcal{F}(x)$ is given by

$$
\mathcal{F}(x)=\frac{x}{1+\zeta x}
$$

with $\zeta=2\left(\gamma^{2} K+m \epsilon \delta\right) /(\gamma \epsilon \delta)$ for (10) and $\zeta=\alpha_{1}^{-1}+\alpha_{2}^{-1}$ for (12).

In general, as one varies engine-specific parameters, such as $\epsilon$ and $\delta$ in our model or $T_{1 i}$ and $T_{2 i}$ in the endoreversible engine, $s_{1}$ and $s_{2}$ will move along a curve $s_{1}=\mathcal{F}\left(s_{2}\right)$. We now address the question whether the function $\mathcal{F}(x)$ in (13) is uniquely determined for all systems displaying the Curzon-Ahlborn efficiency. In Fig. 3, we draw an arbitrary curve (dotted line) in $\left(s_{1}, s_{2}\right)$ plane. The thermodynamic second law $s_{1} \leq s_{2}$ requires that the function $\mathcal{F}(x)$ should be below the straight line $s_{1}=s_{2}$. The device works as a heat engine when $s_{1}>0, s_{2}>0$, and $w=q_{1}+q_{2}=T_{1} s_{1}-T_{2} s_{2} \geq 0$. Hence, the shaded area between two straight lines $s_{1}=s_{2}$ and $s_{1}=\left(T_{2} / T_{1}\right) s_{2}=\left(1-\eta_{C}\right) s_{2}$ is the region of physical interest. Noting that the power of the engine is constant along a straight line $s_{1}=\left(1-\eta_{C}\right) s_{2}+w / T_{1}$, one finds that the maximum power achieved when the curve $s_{1}=\mathcal{F}\left(s_{2}\right)$ is tangential with the straight line of slope $\left(1-\eta_{C}\right)$. The tangential point $\left(s_{1}^{*}, s_{2}^{*}\right)$, hence the maximum power point, is determined by

$$
s_{1}^{*}=\mathcal{F}\left(s_{2}^{*}\right),\left(1-\eta_{C}\right)=\mathcal{F}^{\prime}\left(s_{2}^{*}\right) .
$$

The efficiency at maximum power is then given by

$$
\eta_{M P}=1-\left(1-\eta_{C}\right) \frac{s_{2}^{*}}{s_{1}^{*}} .
$$

We now impose that $\eta_{M P}=1-\sqrt{1-\eta_{C}}$ for any combinations for $T_{1}$ and $T_{2}$, i.e., any value of $\eta_{C}$. Eliminating $\eta_{C}$ using (14) and (15), we obtain the differential equation for the function $\mathcal{F}(x)$ :

$$
\mathcal{F}^{\prime}(x)=\frac{\mathcal{F}(x)^{2}}{x^{2}} .
$$

The solution of the differential equation is given by the function in (13). This analysis shows that the CurzonAhlborn efficiency at maximum power is achieved if and only if the entropy loss rate in the hot reservoir and the entropy gain rate in the cold reservoir are constrained by the function given in (13).

We add a few remarks. Firstly, there have been attempts to understand the Curzon-Ahlborn efficiency from the symmetry consideration. Near equilibrium where $T_{1} \simeq T_{2}$ or $\eta_{C}=1-T_{2} / T_{1} \ll 1$, the CurzonAhlborn efficiency is expanded as $\eta_{C A}=\frac{1}{2} \eta_{C}+\frac{1}{8} \eta_{C}^{2}+$ $O\left(\eta_{C}^{3}\right)$. The first order term $\frac{1}{2} \eta_{C}$ reflects the strong coupling between thermodynamic fluxes [29]. Namely, the heat fluxes and mechanical flux are proportional to each other so that the total entropy production should be also proportional to the heat flux or $s_{1}$. The function form $\mathcal{F}(x)=x /(1+\zeta x)$ implies that the total entropy production rate is given by $s_{t o t}=-s_{1}+s_{2}=\zeta s_{1} s_{2}$, which shows that our model belongs to the strong coupling category. The second order term $\frac{1}{8} \eta_{C}^{2}$ is a manifestation of the so-called left-right symmetry [30] under the exchange of the role between the hot and cold heat baths. 


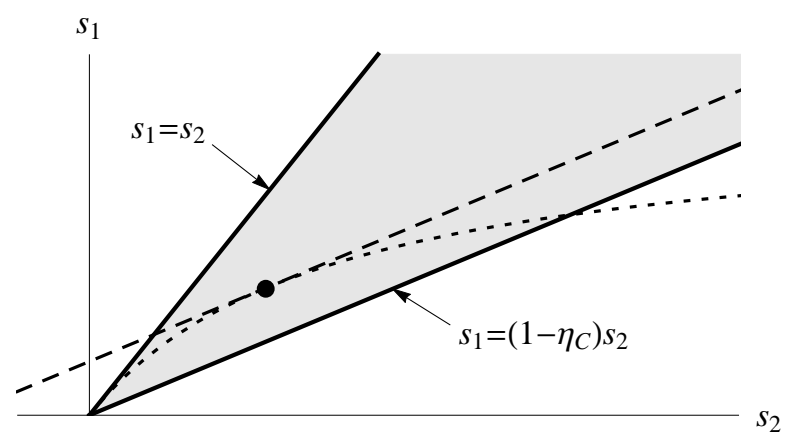

FIG. 3. Linear heat engine model in the $\left(s_{1}, s_{2}\right)$ plane. The model acts as a heat engine in the shaded area satisfying (1$\left.\eta_{C}\right) s_{2}<s_{1}<s_{2}$. The dotted curve represents a characteristic of an engine. The maximum power is achieved when the curve is tangential to a straight line of slope $\left(1-\eta_{C}\right)$.

Note that the relation $s_{1}=\mathcal{F}\left(s_{2}\right)$ is invariant under the changes $s_{1} \rightarrow-s_{2}$ and $s_{2} \rightarrow-s_{1}$ because the inverse of $\mathcal{F}(x)$ is given by $\mathcal{F}^{-1}(x)=-\mathcal{F}(-x)$. Thus, our model has the left-right symmetry. The higher order terms do not have a simple explanation yet. Hopefully, our result may shed some lights in revealing the physical meaning of the whole higher order terms.

Secondly, in general, as one varies microscopic parameters, an engine may cover the whole physical region in the $\left(s_{1}, s_{2}\right)$ plane instead of following a one-dimensional curve such as $s_{1}=\mathcal{F}\left(s_{2}\right)$ in our model. Such a onedimensional representation is possible when there exist only a single independent parameter. The model of Curzon and Ahlborn includes two parameters $T_{1 i}$ and $T_{2 i}[1]$. However, the endoreversibility condition eliminates one degree of freedom. In our model, we reduced the number of independent parameters by following the constant $\epsilon \delta$ curve. For general heat engines with multiple degrees of freedom, if the entropy production rates of two reservoirs satisfy $s_{1}=\mathcal{F}\left(s_{2}\right)$ with a certain parameter $\zeta$, the efficiency at maximum power at constant $\zeta$ is always given by $\eta_{C A}$. It raises questions on the universality of $\eta_{C A}$ and on the role of such a parameter $\zeta$, which are beyond the scope of the present study.

\section{EFFICIENCY FLUCTUATION}

The efficiency $\eta$ is a fluctuating random variable. Recent studies suggest that it is least probable that a nonequilibrium heat engine would achieve the Carnot efficiency $\eta_{C}=1-T_{2} / T_{1}$ in the long time limit [32, 33]. This result is derived for an engine which possesses a finite number of microscopic states and is driven by a time-symmetric protocol. Our engine is driven by a timeindependent protocol which is obviously time-symmetric. However, its phase space is continuous with infinitely many microscopic states. We will examine whether the general statement of Refs. [32, 33] is also valid in our model.
For simplicity, we consider the overdamped dynamics. Hereafter, the time will be rescaled so that the damping coefficient is taken to be unity. Then, the equations of motion for the position vector $\boldsymbol{x}=\left(x_{1}, x_{2}\right)^{T}$ are written as $\dot{\boldsymbol{x}}=\boldsymbol{f}+\boldsymbol{\xi}$ with the force $\boldsymbol{f}=\boldsymbol{f}_{c}+\boldsymbol{f}_{n c}$ and the thermal noise $\boldsymbol{\xi}=\left(\xi_{1}, \xi_{2}\right)^{T}$. Our task is to find the probability distribution $P_{t}(\eta)$ for the stochastic efficiency $\eta=W / Q_{1}$ where $Q_{1}$ is the heat absorbed from the hot reservoir and $W$ is the work done against the nonconservative force $\boldsymbol{f}_{n c}$ up to time $t$ (we will drop the subscript in $Q_{1}$ for notational convenience). We focus on the large deviation function (LDF)

$$
L(\eta) \equiv-\lim _{t \rightarrow \infty} \frac{1}{t} \ln P_{t}(\eta) .
$$

In order to find $P_{t}(\eta)$, one needs to obtain the joint probability distribution $p(Q, W ; t)$. It is accessible by considering the Fokker-Planck equation for the probability distribution $p(\boldsymbol{y} ; t)$ of the four-component vector $\boldsymbol{y}=\left(x_{1}, x_{2}, Q, W\right)^{T}$. This method was introduced for the heat fluctuation of a one-dimensional Brownian particle [46]. We extend the method to calculate the joint distribution for $Q$ and $W$. The generating function is defined as

$$
G_{t}\left(x_{1}, x_{2}, \lambda_{Q}, \lambda_{W}\right) \equiv \int d Q d W e^{-\lambda_{Q} Q-\lambda_{W} W} p(\boldsymbol{y} ; t) .
$$

After a lengthy algebra, we find that

$$
G_{t} \propto \exp \left[-\frac{1}{2} \boldsymbol{x}^{T} \cdot \mathrm{D}^{-1 / 2} \mathrm{JD}^{-1 / 2} \cdot \boldsymbol{x}+\mu\left(\lambda_{Q}, \lambda_{W}\right) t\right]
$$

in the large $t$ limit, where $\mathrm{J}=\mathrm{J}\left(\lambda_{Q}, \lambda_{W}\right)$ is a symmetric $2 \times 2$ matrix and

$$
\mu\left(\lambda_{Q}, \lambda_{W}\right)=H\left(\lambda_{Q}+\bar{\eta} \lambda_{W}\right)
$$

with the function

$$
H(\Lambda)=K-\sqrt{K^{2}+\epsilon^{2} T_{1} T_{2}\left[\Lambda_{m}^{2}-\left(\Lambda-\Lambda_{m}\right)^{2}\right]}
$$

with

$$
\Lambda_{m}=\frac{\left(\eta_{C}-\bar{\eta}\right)}{2 T_{2}} \geq 0
$$

Here, $\bar{\eta}=\langle W\rangle /\langle Q\rangle=1-\delta / \epsilon$ is the average efficiency derived in the previous section and $\eta_{C}=1-T_{2} / T_{1}$ is the Carnot efficiency. The derivation and the exact expression for $\mathrm{J}$ are presented in Appendix $\mathrm{A}$

After integrating $G_{t}\left(x_{1}, x_{2}, \lambda_{Q}, \lambda_{W}\right)$ over $\boldsymbol{x}$, one obtains the reduced generating function $\tilde{G}_{t}\left(\lambda_{Q}, \lambda_{W}\right)$ for $Q$ and $W$. The integration does not introduce an additional $t$-dependent term in the exponent as far as $\mathrm{J}$ is positivedefinite. Therefore, the cumulant generating function $(\mathrm{CGF}) \phi\left(\lambda_{Q}, \lambda_{W}\right)=\lim _{t \rightarrow \infty} \frac{1}{t} \ln \tilde{G}_{t}\left(\lambda_{Q}, \lambda_{W}\right)$ [33] is given by

$$
\phi\left(\lambda_{Q}, \lambda_{W}\right)=\mu\left(\lambda_{Q}, \lambda_{W}\right) \chi_{\mathrm{J}}\left(\lambda_{Q}, \lambda_{W}\right),
$$


where the characteristic function $\chi_{\mathrm{J}}\left(\lambda_{Q}, \lambda_{W}\right)$ is equal to unity if the matrix $\mathrm{J}\left(\lambda_{Q}, \lambda_{W}\right)$ is positive-definite and infinity otherwise.

The LDF $L(\eta)$ is then obtained by using the relation

$$
L(\eta)=-\min _{\lambda} \phi(-\eta \lambda, \lambda)
$$

that was derived in Ref. [33]. To a given value of $\eta$, one need to evaluate the minimum value of the function $\phi\left(\lambda_{Q}, \lambda_{W}\right)$ along a straight line $l_{\eta}$ of slope $-\eta$ passing through the origin in the $\boldsymbol{\lambda}=\left(\lambda_{W}, \lambda_{Q}\right)$ plane. Such a task is achieved by using the property of the function $\mu$. Recall that $\mu\left(\lambda_{Q}, \lambda_{W}\right)=H\left(\lambda_{Q}+\bar{\eta} \lambda_{W}\right)$ depends on a single parameter $\Lambda=\lambda_{Q}+\bar{\eta} \lambda_{W}$. Thus, it is constant along a straight line of slope $-\bar{\eta}$ in the $\boldsymbol{\lambda}$ plane. The function $H(\Lambda)$ has the minimum value

$$
\mu_{m}=K-\sqrt{K^{2}+\epsilon^{2} T_{1} T_{2} \Lambda_{m}^{2}} \leq 0
$$

at $\Lambda=\Lambda_{m}$, and increases monotonically as $\Lambda$ deviates from $\Lambda_{m}$. Thus, $L(\eta)$ is determined by the distance of the line $l_{\eta}$ and $\Lambda=\Lambda_{m}$ inside the domain of $\chi_{\mathrm{J}}=1$.

In Fig. 4 we explain a graphical method to construct $L(\eta)$. This method gives an information on the shape of $L(\eta): L(\eta=\bar{\eta})=0, L(\eta)$ increases monotonically as $\eta$ deviates from $\bar{\eta}$ in the region $\eta_{L}<\eta<\eta_{R}$, and remains constant $L(\eta)=-\mu_{m} \geq 0$ elsewhere. The boundaries $\eta_{L}(\leq \bar{\eta})$ and $\eta_{R}(\geq \bar{\eta})$ vary with model parameters. In Fig. 5 , we show the plot of $L(\eta)$ obtained from the analytic method using the parameters $K=1, T_{1}=2$, $T_{2}=1, \epsilon=1 / 2$, and $\delta=3 / 8$ with the mean efficiency $\bar{\eta}=1-\delta / \epsilon=1 / 4$. The LDF takes the minimum value 0 at $\eta=\bar{\eta}$ and a constant value $-\mu_{m}=(\sqrt{17}-4) / 4$ in the regions with $\eta \leq \eta_{L} \simeq 0.098$ and $\eta \geq \eta_{R} \simeq 0.278$.

We also performed numerical simulations to confirm the analytic result. Starting from the fixed initial configuration $x_{1}=x_{2}=0$, we integrated the time-discretized overdamped Langevin equation numerically by using the Heun method [47] with $\Delta t=0.01$. We measured the work and the heat up to time $t=512,1024$, and 2048, and constructed the probability distribution for the efficiency $P_{t}(\eta)$ by repeating the simulations for $N_{s}=3 \times$ $2^{25} \approx 10^{8}$ times. The LDF $L(\eta)$ can be estimated by fitting $-\frac{1}{t} \ln P_{t}(\eta)$ to the function $A(\eta) / t+B(\eta) \ln t / t+L(\eta)$ at each value of $\eta[48,49]$. The LDF thus obtained is presented in Fig. 5] The numerical result is in good agreement with the analytic result: $L(\eta)$ is minimum at $\eta=\bar{\eta}$ and monotonically increases as $\eta$ deviates from $\bar{\eta}$ to reach its maximum although statistical uncertainty becomes noticeable for large $|\eta-\bar{\eta}|$.

Also shown in Fig. 5 is the LDF obtained from the steady-state initial condition where $x_{1}$ and $x_{2}$ at $t=0$ are drawn from the steady-state distribution. Interestingly, the LDF is different from the LDF obtained from the fixed initial condition. The LDF of nonequilibrium fluctuations may be affected by the initial condition due to the everlasting initial memory effect [50]. Our results exemplify the initial condition dependent behavior of the LDF (see also Appendix A).

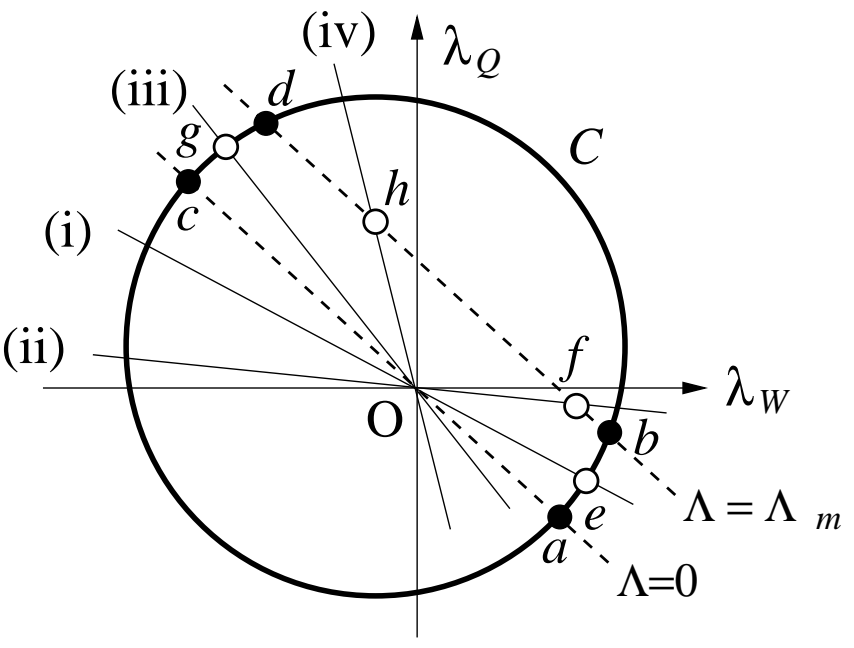

FIG. 4. In the $\boldsymbol{\lambda}=\left(\lambda_{W}, \lambda_{Q}\right)$ plane, we draw schematically the boundary $C$ of the $\left(\chi_{\mathrm{J}}=1\right)$ domain. Two dashed straight lines of slope $-\bar{\eta}$ correspond to $\Lambda=0$ and $\Lambda_{m}$ where $\Lambda=$ $\lambda_{Q}+\bar{\eta} \lambda_{W}$. The intersections between them are marked with closed circles and labeled as $a, b, c$, and $d$. To a given value of $\eta, L(\eta)$ is obtained from the minimum value of $\mu\left(\lambda_{Q}, \lambda_{W}\right)$ along the segment of the straight line $l_{\eta}$ passing through the origin $O$ with slope $-\eta$ inside the boundary $C$. When $\eta=\bar{\eta}$, the line $l_{\eta}$ coincides with the line $\Lambda=0$ where $\mu=0$. Hence, $L(\bar{\eta})=0$. (i) When $\eta_{L} \leq \eta<\bar{\eta}$, the right intersection point $e$ (open symbol) of $l_{\eta}$ and $C$ lies on a segment between $a$ and $b$. Thus, the LDF is determined by the $\Lambda$ value at $e$, $L(\eta)=-H\left(\Lambda_{e}\right)$. The left intersection point is irrelevant since it is farther from the line $\Lambda=\Lambda_{m}$ than $e$. The territory $\eta_{L}$ is determined by the condition that the point $e$ coincides with $b$. (ii) When $\eta<\eta_{L}$, the line $l_{\eta}$ intersects with the line $\Lambda=\Lambda_{m}$ within $C$ at point $f$ (open symbol). Hence, $L(\eta)=-\mu_{m}$. (iii) When $\bar{\eta}<\eta \leq \eta_{R}$, the left intersection point $g$ (open symbol) lies on a segment between $c$ and $d$. Thus, the LDF is given by $L(\eta)=-H\left(\Lambda_{g}\right)$. The territory $\eta_{R}$ is determined by the condition that $g$ coincides with $d$. (iv) When $\eta>\eta_{R}$, the line $l_{\eta}$ intersects with the line $\Lambda=\Lambda_{m}$ at point $h$ (open symbol), and $L(\eta)=-\mu_{m}$.

The LDF of our model system does not follow the universal behavior, suggested in Ref. [32, 33], that the Carnot efficiency is the sole maximum point of the LDF. We explain the reason for this discrepancy. In Ref. [32, 33], the least likeliness of the Carnot efficiency was shown for systems possessing a finite number $\Omega_{\text {sys }}$ of microscopic states. The finiteness of $\Omega_{\text {sys }}$ plays a crucial role. The total entropy production of the engine and two heat baths are given by $\Delta S_{t o t}=-\frac{Q_{1}}{T_{1}}-\frac{Q_{2}}{T_{2}}+\Delta S_{\text {sys }}$ with the Shannon entropy change $\Delta S_{\text {sys }}$ of the system. The energy conservation requires that $\Delta E=Q_{1}+Q_{2}-W$ where $\Delta E$ denotes the change in the internal energy of the engine. Eliminating $Q_{2}$, the total entropy production is given by $\Delta S_{t o t}=\frac{\eta_{C}}{T_{2}} Q_{1}-\frac{1}{T_{2}} W+\left(-\frac{1}{T_{2}} \Delta E+\Delta S_{\text {sys }}\right)$. Note that the mean value of $Q_{1}$ and $W$ increases linearly in $t$. On the other hand, when $\Omega_{\text {sys }}$ is finite, $|\Delta E|$ is bounded above by $\left(E_{\max }-E_{\min }\right)$ with the maximum (minimum) energy among the $\Omega_{\text {sys }}$ states and 


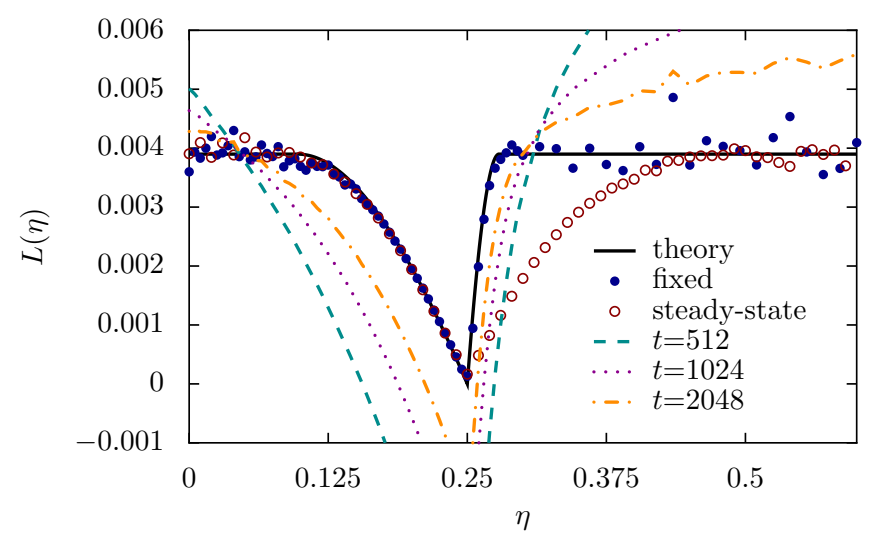

FIG. 5. The LDF for efficiency with the set of parameters $K=1, T_{1}=2, T_{2}=1, \epsilon=1 / 2$, and $\delta=3 / 8$. Black line represents the analytic result obtained from Eq. (24). Filled circles represent the numerical results from the fixed initial condition, while open circles represent those from the steadystate initial condition. The colored lines represent $-\frac{1}{t} \ln P_{t}(\eta)$ at $t=512,1024$, and 2048. Details for the simulations are explained in the main text.

$\left|\Delta S_{\text {sys }}\right|$ by $\ln \Omega_{\text {sys }}$. Consequently, $\Delta S_{\text {sys }}$ may be approximated as $\Delta S_{\text {tot }} \simeq \frac{\eta_{C}}{T_{2}} Q_{1}-\frac{1}{T_{2}} W$ in the large $t$ limit. When the total entropy production is written as the sum of thermodynamic quantities, the joint probability distribution of them satisfies the fluctuation theorem [51 54]

$$
\frac{P\left(Q_{1}, W\right)}{P\left(-Q_{1},-W\right)} \simeq e^{-\Delta S_{t o t}}
$$

The least likeliness of the Carnot efficiency is the direct consequence of the fluctuation theorem [32, 33].

In contrast to the underlying assumption of Refs. 32, 33], our model has the continuous phase space and the internal energy of the engine is unbounded. Although the averages of $\Delta S_{\text {sys }}$ and energy $\Delta E$ are zero in the steady state, stochastic fluctuations may generate rare events accompanied by $\Delta S_{s y s}$ and $\Delta E$ comparable with $Q_{i}$ and $W[55]$. It is known that such fluctuations are nonnegligible and invalidate the fluctuation theorem derived by ignoring them [53, 54, 56]. Therefore, we conclude that the least likeliness of the Carnot efficiency based on the fluctuation theorem in (26) is not valid in our model.

\section{SUMMARY AND DISCUSSIONS}

In this paper, we introduced a model for a heat engine which operates between two heat baths and is driven by a nonconservative force. The model is described by a Ornstein-Uhlenbeck process and most of the properties are analytically tractable. Firstly, we showed that the efficiency at maximum power is given by the so-called Curzon-Ahlborn efficiency $\eta_{M P}=\eta_{C A}=1-\sqrt{T_{2} / T_{1}}$. This is a surprising result because $\eta_{C A}$ has been believed to be the property of the endoreversible engine while our engine is not endoreversible. Instead, we showed that $\eta_{C A}$ is the consequence of the relation $s_{1}=\mathcal{F}\left(s_{2}\right)$ between the entropy loss $s_{1}$ of the hot bath and the entropy gain $s_{2}$ of the cold bath with the universal function given in (13).

Secondly, we derived the analytic expression for the LDF $L(\eta)$ of the efficiency fluctuation. The shape of $L(\eta)$ is shown in Fig. 55 It is minimum at $\eta=\bar{\eta}$ and displays plateaus far from $\bar{\eta}$. Our result shows that $L(\eta)$ does not have a peak at the Carnot efficiency $\eta_{C}$. Thus, the least likeliness of the Carnot efficiency is limited to systems only with a finite number of microscopic states. Nonequilibrium fluctuations in systems with continuous degrees of freedom invalidate the least likeliness of $\eta_{C}$. We also found that the LDF of the efficiency depends on the initial condition, which stresses the initial memory effect of nonequilibrium systems [50].

The linear solvable model has provided a lot of informations on the properties of the heat engines. It also suggests interesting theoretical questions. It is shown that the the Curzon-Ahlborn efficiency at maximum power is guaranteed by the relation $s_{1}=\mathcal{F}\left(s_{2}\right)$ with the universal function $\mathcal{F}(x)$ given in (13). On the other hand, the Curzon-Ahlborn efficiency was investigated from the viewpoint of symmetry in Refs. 29 31]. It would be interesting to pursue the implication of the relation $s_{1}=$ $\mathcal{F}\left(s_{2}\right)$ on underlying symmetry of engine dynamics. The LDF $L(\eta)$ for the system under the steady-state initial condition requires the whole eigenstates of the FokkerPlanck operator, which are not available yet. We would like to leave those tasks for future works.

\section{ACKNOWLEDGMENTS}

This research was supported by the National Research Foundation (NRF) of Korea Grant No. 2013R1A2A2A05006776.

\section{Appendix A: Derivation of $L(\eta)$ and discussion on the initial condition dependency}

In the overdamped limit, the infinitesimal heat and work in (4) during the time interval $d t$ are given by $d Q_{1}=$ $-f_{1} \circ d x_{1}=-f_{1}^{2} d t-f_{1} \circ d \Xi_{1}$ and $d W=-f_{n c} \circ d \boldsymbol{x}=$ $-\left(\boldsymbol{f}_{n c} \cdot \boldsymbol{f}\right) d t-f_{n c, 1} \circ d \Xi_{1}-f_{n c, 2} \circ d \Xi_{2}$ ( $\gamma$ is set to 1$)$. We will use $\cdot$ for the inner product of a vector with another vector or a matrix. Hence, $\boldsymbol{y}=\left(x_{1}, x_{2}, Q=Q_{1}, W\right)^{T}$ follows a stochastic differential equation

$$
\dot{\boldsymbol{y}}=\boldsymbol{d}+\mathrm{N} \cdot \boldsymbol{\zeta},
$$

where the drift vector $\boldsymbol{d}=\left(f_{1}, f_{2},-f_{1}^{2},-\boldsymbol{f} \cdot \boldsymbol{f}_{n c}\right)^{T}$, the $(4 \times 2)$ noise matrix $\mathrm{N}$ is given by

$$
\mathbf{N}=\left(\begin{array}{cc}
\sqrt{2 T_{1}} & 0 \\
0 & \sqrt{2 T_{2}} \\
-\sqrt{2 T_{1}} f_{1} & 0 \\
-\sqrt{2 T_{1}} f_{n c, 1} & -\sqrt{2 T_{2}} f_{n c, 2}
\end{array}\right),
$$


and the components of the noise vector $\boldsymbol{\zeta}(t)=$ $\left(\zeta_{1}(t), \zeta_{2}(t)\right)^{T}$ are independent Gaussian random variables of zero mean and unit variance. The total force $\boldsymbol{f}=\boldsymbol{f}_{c}+\boldsymbol{f}_{n c}$ is linear in $\boldsymbol{x}$, hence it is written as $\boldsymbol{f}=-\mathrm{F} \cdot \boldsymbol{x}$ with the force matrix $F$.

The differential equation is nonlinear and involves the multiplicative noises implemented with the Stratonovich interpretation. Following the standard recipe [39], one can derive the Fokker-Planck equation for the probability distribution $p(\boldsymbol{y} ; t)$ :

$$
\frac{\partial p}{\partial t}=\mathcal{L} p
$$

where the Fokker-Planck operator is given by

$$
\mathcal{L}=-\boldsymbol{\nabla}^{T} \cdot \boldsymbol{d}+\frac{1}{2}\left(\boldsymbol{\nabla}^{T} \cdot \mathrm{N}\right) \cdot\left(\boldsymbol{\nabla}^{T} \cdot \mathrm{N}\right)^{T}
$$

with the differential operator $\nabla=\left(\frac{\partial}{\partial x_{1}}, \frac{\partial}{\partial x_{2}}, \frac{\partial}{\partial Q}, \frac{\partial}{\partial W}\right)^{T}$.

For $G\left(x_{1}, x_{2}, \lambda_{Q}, \lambda_{W} ; t\right)$ defined in (18), the time evolution operator $\mathcal{L}_{\lambda}$ is obtained by replacing $\partial / \partial Q$ and $\partial / \partial W$ in $\mathcal{L}$ with $\lambda_{Q}$ and $\lambda_{W}$, respectively. The resulting operator becomes bilinear in $\boldsymbol{x}$ and the gradient operator $\boldsymbol{\nabla}_{\boldsymbol{x}}=\left(\frac{\partial}{\partial x_{1}}, \frac{\partial}{\partial x_{2}}\right)^{T}$, i.e.,

$$
\mathcal{L}_{\lambda}=\boldsymbol{\nabla}_{\boldsymbol{x}}^{T} \cdot \mathrm{D} \cdot \boldsymbol{\nabla}_{\boldsymbol{x}}+2 \boldsymbol{x}^{T} \cdot \mathrm{B}^{T} \cdot \boldsymbol{\nabla}_{\boldsymbol{x}}+\boldsymbol{x}^{T} \cdot \mathrm{A} \cdot \boldsymbol{x}+K+\operatorname{Tr} \mathrm{B},
$$

where $\mathrm{A}$ and $\mathrm{D}=\operatorname{diag}\left(T_{1}, T_{2}\right)$ are the $2 \times 2$ symmetric matrices, $B$ is the $2 \times 2$ nonsymmetric matrix, and $\operatorname{Tr} X$ denotes the trace of a matrix $X$. The matrix elements for $A$ and $B$ are readily read from A4 . Explicitly, they are given by $\mathrm{A}=\mathrm{C}^{T} \mathrm{DC}+\frac{1}{2} \mathrm{~F}^{T} \mathrm{C}+\frac{1}{2} \mathrm{C}^{T} \mathrm{~F}$ and $\mathrm{B}=\mathrm{DC}+\frac{1}{2} \mathrm{~F}$ with an auxiliary matrix

$$
\mathbf{C}=\left(\begin{array}{cc}
K \lambda_{Q} & -\epsilon\left(\lambda_{Q}+\lambda_{W}\right) \\
-\delta \lambda_{W} & 0
\end{array}\right)
$$

We now rescale the coordinates to define $\hat{\boldsymbol{x}}=$ $\left(\hat{x}_{1}, \hat{x}_{2}\right)^{T} \equiv \mathrm{D}^{-1 / 2} \cdot \boldsymbol{x}$. Then, the time evolution operator is rewritten in terms of $\hat{\boldsymbol{x}}$ as

$$
\mathcal{L}_{\lambda}=\nabla_{\hat{\boldsymbol{x}}}^{2}+2 \hat{\boldsymbol{x}}^{T} \cdot \hat{\mathrm{B}}^{T} \cdot \nabla_{\hat{\boldsymbol{x}}}+\hat{\boldsymbol{x}}^{T} \cdot \mathrm{DA} \cdot \hat{\boldsymbol{x}}+K+\operatorname{Tr} \mathrm{B}
$$

where $\nabla_{\hat{\boldsymbol{x}}}=\left(\frac{\partial}{\partial \hat{x}_{1}}, \frac{\partial}{\partial \hat{x}_{2}}\right)^{T}$ and $\hat{X}=D^{-1 / 2} \cdot \mathrm{X} \cdot \mathrm{D}^{1 / 2}$ for any matrix $X$. It looks similar to the Hamiltonian of the two-dimensional harmonic oscillator except for the second term. Finally, we make a transformation

$$
\widetilde{\mathcal{L}}_{\lambda} \equiv e^{\frac{1}{2} \hat{\boldsymbol{x}}^{T} \cdot \mathrm{J} \cdot \hat{\boldsymbol{x}}} \mathcal{L}_{\lambda} e^{-\frac{1}{2} \hat{\boldsymbol{x}}^{T} \cdot \mathrm{J} \cdot \hat{\boldsymbol{x}}}
$$

with a certain symmetric matrix $J$ which will be determined later. It acts as the time evolution operator for the modified generating function $e^{\frac{1}{2} x^{T} \cdot \mathrm{D}^{-1 / 2} \mathrm{JD}^{-1 / 2} \cdot \boldsymbol{x}} G\left(\boldsymbol{x}, \lambda_{Q}, \lambda_{W} ; t\right)$. This transformation replaces the gradient operator $\nabla_{\hat{\boldsymbol{x}}}$ with $\boldsymbol{\nabla}_{\hat{\boldsymbol{x}}}-\mathrm{J} \cdot \hat{\boldsymbol{x}}$, which leads to

$$
\widetilde{\mathcal{L}}_{\lambda}=\nabla_{\hat{\boldsymbol{x}}}^{2}-2 \hat{\boldsymbol{x}}^{T} \cdot \mathrm{M}^{T} \cdot \nabla_{\hat{\boldsymbol{x}}}+\hat{\boldsymbol{x}}^{T} \cdot \mathrm{Q} \cdot \hat{\boldsymbol{x}}+\mu,
$$

where

$$
\begin{aligned}
& \mathrm{M}=\mathrm{J}-\hat{\mathrm{B}}, \\
& \mathrm{Q}=\mathrm{M}^{T} \mathrm{M}+\mathrm{D} \hat{\mathrm{A}}-\hat{\mathrm{B}}^{T} \hat{\mathrm{B}}=\mathrm{M}^{T} \mathrm{M}-\frac{1}{4} \hat{\mathrm{F}}^{T} \hat{\mathrm{F}}, \\
& \mu=K-\operatorname{Tr} \mathrm{M} .
\end{aligned}
$$

The operator $\widetilde{\mathcal{L}}_{\lambda}$ is simplified if one chooses $\mathrm{J}$ or $\mathrm{M}$ suitably so that $Q=0$. It is accomplished by choosing

$$
\mathrm{J}=\hat{\mathrm{B}}+\frac{1}{2} \mathrm{O} \hat{\mathrm{F}}
$$

with an orthogonal matrix

$$
\mathrm{O}=\left(\begin{array}{cc}
\cos \theta & -\sin \theta \\
\sin \theta & \cos \theta
\end{array}\right)
$$

The angle variable $\theta$ has to be determined by requiring that $J$ should be a symmetric matrix. Then, the time evolution operator $\widetilde{\mathcal{L}}_{\lambda}$ has the constant eigenfunction with the corresponding eigenvalue $\mu$. As a result, in the large $t$ limit, the generating function $G$ has the asymptotic form in (19).

We find that the symmetry condition $J_{12}=J_{21}$ is satisfied if

$$
\theta=\alpha \pm \beta
$$

where $\cos \alpha=\frac{\hat{F}_{12}-\hat{F}_{21}}{R}, \sin \alpha=-\frac{\hat{F}_{11}+\hat{F}_{22}}{R}, \cos \beta=$ $\frac{2\left(\hat{B}_{21}-\hat{B}_{12}\right)}{R}$, and $\sin \beta=\sqrt{1-\cos ^{2} \beta}$ with

$$
R=\sqrt{\left(\hat{F}_{12}-\hat{F}_{21}\right)^{2}+\left(\hat{F}_{11}+\hat{F}_{22}\right)^{2}}
$$

There are two different solutions for $\mathrm{J}$ due to the sign ambiguity in (A13). To select the proper solution, we impose the condition that the generating function $G\left(\boldsymbol{x}, \lambda_{Q}, \lambda_{W} ; t\right)$ in the infinite $t$ limit should converge to the steady-state distribution when $\lambda_{Q}=\lambda_{W}=0$. The steady-state probability distribution of a linear system is known exactly 35]. Comparing the two solutions with the steady-state probability distribution, we find that the matrix $\mathrm{J}$ is indeed given by $\mathrm{J}=\hat{\mathrm{B}}+\frac{1}{2} \mathrm{O} \hat{\mathrm{F}}$ with $\theta=\alpha+\beta$. The eigenvalue is given by

$$
\mu\left(\lambda_{Q}, \lambda_{W}\right)=K-\frac{R}{2} \sin \beta,
$$

which yields the result in (20).

We add a remark on the initial condition dependence. In our calculation, we keep only the leading eigenvalue $\mu$ of $\widetilde{\mathcal{L}}_{\lambda}$. Integration over the final position $\boldsymbol{x}$ introduces a cut represented by the characteristic function $\chi_{\mathrm{J}}$ to yield the result in (23). As for the effect of the initial condition, Visco studied a similar problem, a Brownian particle in one dimension in contact with two heat baths [46]. Visco obtained the exact moment generating functions for both the fixed and the steady-state initial conditions by considering all the eigenstates of the Fokker-Planck operator. 
The study reveals that fluctuations in the initial configuration introduce an additional characteristic function. In this regard, we expect that the steady-state initial condition in our model modifies the characteristic function $\chi_{\mathrm{J}}$ so that the LDF $L(\eta)$ in the steady-state initial condition is broader. We confirm this expectation with numerical simulations. In Fig. 5, we compare the LDFs from the fixed initial condition and from the steady-state initial condition. One finds that the LDF from the latter displays a broader distribution in the $\eta \geq \bar{\eta}$ side although the analytic expression for that is not available yet.
[1] F. L. Curzon and B. Ahlborn, Am. J. Phys. 43, 22 (1975).

[2] P. Chambadal, Les Centrales Nucléaires (Armand Colin, 1957).

[3] I. I. Novikov, J. Nuclear Energy II 7, 125 (1958).

[4] D. P. Sekulic, J. Appl. Phys. 83, 4561 (1998).

[5] B. Andersen, J. Appl. Phys. 90, 6557 (2001).

[6] A. Gomez-Marin and J. M. Sancho, Phys. Rev. E 74, 062102 (2006).

[7] T. Schmiedl and U. Seifert, Europhys. Lett. 81, 20003 (2008).

[8] Z. C. Tu, J. Phys. A: Math. Theor. 41, 312003 (2008).

[9] Y. Izumida and K. Okuda, Europhys. Lett. 83, 60003 (2008).

[10] Y. Izumida and K. Okuda, Phys. Rev. E 80, 021121 (2009).

[11] Y. Izumida and K. Okuda, Prog. Theor. Phys. Suppl. 178, 163 (2009).

[12] M. Esposito, K. Lindenberg, and C. Van den Broeck, Europhys. Lett. 85, 60010 (2009).

[13] M. Esposito, R. Kawai, and K. Lindenberg, Phys. Rev. E 81, 041106 (2010).

[14] M. Esposito, N. Kumar, K. Lindenberg, and C. Van den Broeck, Phys. Rev. E 85, 031117 (2012).

[15] Y. Apertet, H. Ouerdane, C. Goupil, and P. Lecoeur, Phys. Rev. E 85, 031116 (2012).

[16] Y. Apertet, H. Ouerdane, C. Goupil, and P. Lecoeur, Phys. Rev. E 85, 041144 (2012).

[17] C. Van den Broeck and K. Lindenberg, Phys. Rev. E 86, 041144 (2012).

[18] T. Schmiedl and U. Seifert, Europhys. Lett. 83, 30005 (2008)

[19] Y. Zhou and D. Segal, Phys. Rev. E 82, 011120 (2010).

[20] U. Seifert, Phys. Rev. Lett. 106, 020601 (2011).

[21] N. Golubeva, A. Imparato, and L. Peliti, Europhys. Lett. 97, 60005 (2012).

[22] C. Van den Broeck, N. Kumar, and K. Lindenberg, Phys. Rev. Lett. 108, 210602 (2012).

[23] N. Golubeva and A. Imparato, Phys. Rev. Lett. 109, 190602 (2012).

[24] N. Golubeva and A. Imparato, Phys. Rev. E 88, 012114 (2013).

[25] H. Hooyberghs, B. Cleuren, A. Salazar, and J. O. Indekeu, J. Chem. Phys. 139, 134111 (2013).

[26] N. Golubeva and A. Imparato, Phys. Rev. E 89, 062118 (2014).

[27] A. E. Allahverdyan, Phys. Rev. E 77, 041118 (2008).

[28] S. Abe, Phys. Rev. E 83, 041117 (2011).

[29] C. Van den Broeck, Phys. Rev. Lett. 95, 190602 (2005).

[30] M. Esposito, K. Lindenberg, and C. Van den Broeck, Phys. Rev. Lett. 102, 130602 (2009).
[31] B. Cleuren, B. Rutten, and C. Van den Broeck, arXiv (2015), 1503.02453v1.

[32] G. Verley, M. Esposito, T. Willaert, and C. Van den Broeck, Nature Communications 5, 1 (2014).

[33] G. Verley, T. Willaert, C. Van den Broeck, and M. Esposito, Phys. Rev. E 90, 052145 (2014).

[34] C. Kwon, P. Ao, and D. Thouless, Proc. Nat. Acad. Sci. 102, 13029 (2005).

[35] C. Kwon, J. D. Noh, and H. Park, Phys. Rev. E 83, 061145 (2011).

[36] J. D. Noh, C. Kwon, and H. Park, Phys. Rev. Lett. 111, 130601 (2013).

[37] H.-M. Chun and J. D. Noh, Phys. Rev. E 91, 052128 (2015).

[38] K. Sekimoto, Prog. Theor. Phys. Suppl. 130, 17 (1998).

[39] H. Risken, The Fokker-Planck Equation, Methods of Solution and Applications (Springer, 1996).

[40] C. Gardiner, Stochastic Methods, A Handbook for the Natural and Social Sciences (Springer, 2010).

[41] Z. Rieder, J. Math. Phys. 8, 1073 (1967).

[42] T. Tomé and M. J. de Oliveira, Phys. Rev. E 82, 021120 (2010).

[43] E. Lippiello, M. Baiesi, and A. Sarracino, Phys. Rev. Lett. 112, 140602 (2014).

[44] A. De Vos, Am. J. Phys. 53, 570 (1985).

[45] A. Bejan, Int. J. Heat Mass Transfer. 31, 1211 (1988).

[46] P. Visco, J. Stat. Mech.: Theor. Exp. 2006, P06006 (2006).

[47] A. Greiner, W. Strittmatter, and J. Honerkamp, J. Stat. Phys. 51, 95 (1988).

[48] K. Proesmans, B. Cleuren, and C. Van den Broeck, Europhys. Lett. 109, 20004 (2015).

[49] K. Proesmans and C. Van den Broeck, New J. Phys. 17, 065004 (2015).

[50] J. S. Lee, C. Kwon, and H. Park, Phys. Rev. E 87, 020104 (2013).

[51] R. García-García, V. Lecomte, A. Kolton, and D. Domínguez, J. Stat. Mech.: Theor. Exp. 2012, P02009 (2012).

[52] R. García-García, D. Domínguez, V. Lecomte, and A. B. Kolton, Phys. Rev. E 82, 030104 (2010).

[53] J. D. Noh and J.-M. Park, Phys. Rev. Lett. 108, 240603 (2012).

[54] J. D. Noh, J. Stat. Mech.: Theor. Exp. 2014, P01013 (2014).

[55] T. Nemoto, Phys. Rev. E 85, 061124 (2012).

[56] R. Van Zon and E. Cohen, Phys. Rev. Lett. 91, 110601 (2003). 